\title{
Evaluation of the Correlation Between Depression and Physical Activity Among Elderly Patients with Osteoporosis: A Cross-Sectional Study
}

\section{Linjun Shi}

West China School of Nursing/Department of International Medical Center, West China Hospital,

Sichuan University

\section{Xuexue Deng}

West China School of Nursing/Department of International Medical Center, West China Hospital,

Sichuan University

\section{Xiaoping Zhou}

West China School of Nursing/Department of International Medical Center, West China Hospital,

Sichuan University

\section{Wenjing Liu}

West China School of Nursing/Department of International Medical Center, West China Hospital,

Sichuan University

\section{Yueshan Gao}

West China School of Nursing, Sichuan University

Ronghua Fang ( $\square$ fangronghua@126.com )

West China School of Nursing/Department of International Medical Center, West China Hospital, Sichuan University

\section{Research Article}

Keywords: anxiety, depression, osteoporosis, elderly, physical activity

Posted Date: July 26th, 2021

DOl: https://doi.org/10.21203/rs.3.rs-687721/v1

License: (c) (1) This work is licensed under a Creative Commons Attribution 4.0 International License. Read Full License 


\section{Abstract}

Background: Depression and osteoporosis are two chronic diseases that affect many people and have a significant impact on incidence, mortality, and quality of life. An association between osteoporosis and depression has been described in the literature. However, few studies have reported the correlation between depression and physical activity in elderly patients with osteoporosis in China.

Methods: This cross-sectional study was conducted from August 1, 2020, to January 29, 2021. Eligible participants were aged $\geq 60$ years and identified as having osteoporosis. One hundred and seventy-three patients were recruited in the outpatient department of West China Hospital. A self-designed demographic data questionnaire, self-rating anxiety scale (SAS), self-rating depression scale (SDS), and physical activity scale in the elderly (PASE) were used to evaluate anxiety and depression status and physical activity of the participants. Multivariate linear regression was performed to evaluate the factors affecting physical activity. Spearman's correlation coefficient was used to analyze the correlation among physical activity, anxiety, and depression in elderly patients with osteoporosis.

Results: Of the 173 participants with osteoporosis, 114 (65.9\%) were aged 60-75 years. The physical activity scores were $78.17 \pm 3.03 .131(75.72 \%)$ for participants with anxiety and $122(70.52 \%)$ for participants with depression. Multiple linear regression showed that age, education, smoking, participation in social activity, activity of daily life, frailty phenotype, SAS, and SDS were independent risk factors for physical activity in elderly patients with osteoporosis. Physical activity was negatively correlated with anxiety and depression. This means that the higher the physical activity score was, the lower the incidence of anxiety and depression.

Conclusions: We found that anxiety and depression were prevalent, and physical activity was negatively correlated with anxiety and depression in elderly patients with osteoporosis in China. Medical staff should focus on the factors that influence anxiety and depression among elderly patients with osteoporosis and reduce the incidence of anxiety and depression.

\section{Background}

China is rapidly becoming an aging society. Older individuals often suffer from osteoporosis (OP) and depression. OP is a common bone disease characterized by low bone density associated with aging, and morbidity increases with age [1]. The pathology is associated with high socioeconomic stress and mortality $[2,3]$.

Epidemiological data reported that the population with OP would increase sharply from 83.9 million in 1997 to 212 million, the number of patients with osteoporosis fractures will increase from 1.63 million to 5.99 million, and the corresponding medical expenditure will be as high as 254 hundred million dollars by 2050 in China [4, 5]. The prevalence of OP in the general population aged 60 years or more in China, has been reported to be $36 \%$, at $49 \%$ in women and $23 \%$ in men [6]. Approximately 9 million osteoporosis or fragility (low-trauma) fractures occur worldwide per year [7]. Approximately $30 \%$ of women will be 
diagnosed with OP, and approximately half of them will require physician-supervised treatment after a fragility fracture $[8,9]$. Only $2 / 3$ of patients with osteoporosis have detected disease, and less than half of those actually receive effective anti-OP drugs $[10,11]$. Physical activity (PA) plays a crucial role in OP, mainly by reducing bone resorption, increasing muscle stability, strength, balance, and mobility, reducing pain and preventing falls, improving quality of life [12,13], and preventing OP and fragility fractures [1416]. Epidemiological data have reported that anxiety and depression influence the older population at a rate of $47.2-49.9 \%$ in China and $30-40 \%$ worldwide [17]. Depression was reported by $69.1 \%$ of osteoporotic elderly people in the CODE study [18] and by $33.0 \%$ of female patients with OP in Germany [19]. Thus far, China has no relevant reports.

This study aims to evaluate the correlation between depression and physical activity among elderly patients with OP.

\section{Methods}

\section{Study design}

Between August 1, 2020, and January 29, 2021, a cross-sectional study using questionnaires was performed among patients with OP in a general hospital in Chengdu, China. The Ethics Committee of West China Hospital of Sichuan University approved this research. Informed consent was obtained from all participants. Survey data were anonymously coded to ensure that identifying information remained confidential.

All study participants had OP diagnoses consistent with the Chinese guidelines for the diagnosis and treatment of senile OP (2018 edition) [20]. The inclusion criteria were as follows: outpatients specializing in endocrinology and osteoporosis, age $\geq 60$ years, diagnosis of OP (bone density examination through dual-energy X-ray,DEXA) [20], and informed and voluntary participation in the survey. The exclusion criteria were as follows: malignant diseases, thyroid, parathyroid and adrenal gland corticosteroids, hormones, and anxiety and depression history.

For data collection, four questionnaires were given to each participant: a self-designed demographic data questionnaire, self-rating anxiety scale (SAS), self-rating depression scale (SDS), and physical activity scale for the elderly (PASE). Members of the research team interviewed each participant in the outpatient department of West China Hospital, Sichuan University. The investigator explained the purpose, significance, and relevant instructions and obtained the signed consent form before administering the survey. The researchers gave the participants a paper questionnaire, the respondents completed the questionnaire, and then the researchers collected it on the spot.

The database was built using EpiData 3.1 software (EpiData-Comprehensive Data Management and Basic Statistical Analysis System, EpiData Association, Odense, Denmark) and then double-checked. Incomplete questionnaires ( $>20 \%$ missing data) were deleted. 


\section{Demographic Data}

This questionnaire was self-designed after a literature review. The participants provided information about their age, gender, education background, marital status, income, number of nonosteoporosis (nonOP) chronic conditions, history of fracture, smoking, drinking, type of drugs, participation in social activity, body mass index (BMI), activity of daily life (ADL) [21] and Fried's frailty phenotype [22].

\section{Self-rating Anxiety Scale (SAS)}

The anxiety level was assessed using Zung's self-rating anxiety scale (SAS), a validated 20 -item selfreport instrument [23]. Cronbach's a was 0.897, and the internal correlation was 0.913 [24]. The instrument employs a four-point Likert scale where " 1 = never or very rare", " 2 = sometimes", " 3 = often", and " 4 = very often or always". Questions 1-5 characterize the emotional points of anxiety, whereas questions 6-20 signify the physical symptoms of anxiety. For each respondent, the sum of the scores for 20 items ranged from 20 to 80 . The sum of scores is then converted to an "Anxiety Index" with values ranging from 25 to 100. Following the recommendations from Zung [25] and Dunstan and Scott [26], mild anxiety: 50-59; moderate anxiety: 60-69; severe anxiety: $\geq 70$. Higher scores reflect worse symptoms.

\section{Self-rating Depression Scale (SDS)}

Symptoms of depression were assessed with Zung's self-rating depression scale (SDS). The self-report questionnaire was developed by Zung [27] to quantify symptoms of depression by using criteria for a prolonged sense of depression and the accompanying physiological and psychological symptoms. The questionnaire includes 20 items, ten of which have a positive symptomatic value and ten of which have a negative symptomatic value. Mild depression: 50-59; moderate depression: 60-69; severe depression: $\geq 70$. Higher scores indicate worse symptoms. The questionnaire has good coefficients of reliability and validity [28].

\section{Physical Activity Scale in the Elderly}

The Chinese version of the physical activity scale in the elderly (PASE) was used to assess participants. The PASE was developed by Washburn et al. and Hu et al. [29, 30]. The questionnaire is self-administered and includes frequency, intensity, and duration of time spent on leisure activity, household chores, and work/volunteer activity over the preceding week. Self-reported PASE scores are well validated against objective measures of activity. The Chinese version of the short International Physical Activity Questionnaire (IPAQ-S-C) has good reliability, and Cronbach's a was 0.725 [31]. PASE scores are calculated from weights and frequency values for each of the 12 types of activity. The total score is from 0 to 400 or more. Higher scores indicate greater physical activity.

\section{Statistical Analysis}

SPSS software (Version 20.0. IBM Inc, Armonk, NY) was used for the statistical analyses. Quantitative data, such as the SAS score, SDS score, and PASE score, are presented as the arithmetic mean and standard deviation. Qualitative data, such as the proportion of participants with anxiety and depression, 
are represented by the percentage. Multivariate linear regression was performed to analyze factors affecting physical activity. Spearman analysis was performed to assess the correlation among PA, SAS, and SDS in patients with OP. A $p$-value $<0.05$ was considered statistically significant.

\section{Results}

\section{Participants}

The data from 207 patients with OP who were $>60$ years old were analyzed, with 173 effective questionnaires, the rate of validity for this study was $83.57 \%$. The 173 participants ranged from 60 to 94 years of age, with an average age of $70.63 \pm 9.02$ years. The sample also had the following characteristics: $60-74$ years, $65.9 \%$, female, $83.2 \%$, married, $79.8 \%$, junior high school and below, $63.0 \%$, living with children, $54.9 \%$.

\section{Demographic characteristics of the participants, anxiety, and depression}

The primary features of the participants and rates of anxiety and depression are summarized in Table 1. A total of 131 (75.72\%) participants had anxiety, and 122 (70.52\%) participants had depression.There were statistically significant differences between anxiety and depression in elderly patients with OP across the following variables: occupation, residence, pain, type of drugs, number of nonOP, chronic conditions, participation in social activity, ADLs, and frailty phenotype. However,income only affected depression $(P \square 0.05)$, and fracture only affected anxiety for patients with OP $(P \otimes 0.05)$ in this study. Thus, the following factors led to higher anxiety and depression and higher SAS and SDS scores among elderly outpatients with OP: no occupation, living with children, pain, one nonOP chronic condition, $\square 5$ types of drugs, no participation in social activity, mild ADLs, and frailty. No significant differences were observed for the other variables (Table 1).

\section{Demographic characteristics of the participants and physical activity}

The basic characteristics of the participants and their physical activity are summarized in Table 2 . The PASE scores of the 173 elderly patients with OP were $78.17 \pm 3.03$. Statistically significant differences were found in demographic characteristics of the participants and physical activity across the following variables: age, marital status, education, occupation, residence, income, pain, type of drugs, participation in social activity, ADLs, frailty phenotype, anxiety, and depression ( $P \otimes 0.05)$. Thus, participants with age $\geq 75$ years, divorced/widowed, primary school and below, no occupation, living with children, child support, pain, $\geq 5$ types of drugs, no participation in social activity, heavy ADLs, frailty, severe anxiety, and severe depression had lower PASE scores. No significant differences were observed for the other variables (Table 2).

\section{Multiple linear regression analysis of factors related to physical activity}

Linear regression results showed that age, education, smoking, participation in social activity, ADLs, frailty phenotype, SAS, and SDS were the main risk factors that affected PA in elderly patients with OP 
(Table 3).

Table 3. Multiple linear regression analysis of factors related to physical activity

\begin{tabular}{|llllll|}
\hline Independent variable & \multicolumn{2}{l}{$\begin{array}{l}\text { Unstandardized } \\
\text { coefficients }\end{array}$} & $\begin{array}{l}\text { Standardized } \\
\text { regression coefficient }\end{array}$ & & \\
\cline { 2 - 5 } & $\beta$ & \multicolumn{2}{c|}{ Standard error } & & \\
Constant & & & & & \\
\hline Age & -29.322 & 8.220 & -0.198 & -3.567 & 0.000 \\
\hline Education & 7.917 & 3.505 & 0.120 & 2.259 & 0.025 \\
\hline Smoking & -42.036 & 12.329 & -0.173 & -3.410 & 0.001 \\
\hline Participation in social activity & 24.871 & 4.890 & 0.272 & 5.086 & 0.000 \\
\hline ADLs & 20.331 & 5.832 & 0.229 & 3.486 & 0.001 \\
\hline Frailty phenotype & -20.751 & 7.022 & -0.182 & -2.955 & 0.004 \\
\hline SAS & 12.316 & 6.052 & 0.183 & 2.035 & 0.043 \\
\hline SDS & -21.697 & 5.735 & -0.359 & -3.784 & 0.000 \\
\hline
\end{tabular}

Analysis of the correlation between physical activity, anxiety, and depression The results of the correlation analysis showed that physical activity was negatively correlated with anxiety and depression (R: SAS = $0.354, \operatorname{SDS}=-0.510, P \otimes 0.05$ ). This means that the higher the PA score was, the lower the incidence of anxiety and depression (Fig. 1).

\section{Discussion}

The present study showed that anxiety and depression were prevalent among elderly outpatients with OP in China. This prevalence is higher than in previous studies $[18,19,32]$. However, our study indicated that participants who were 60-74 years old had a higher prevalence of anxiety and depression (Table 1). Additionally, we found a higher risk of anxiety and depression among individuals with no occupation, living with children, pain, one of the nonOP chronic conditions, $<5$ types of drugs, no participation in social activity, moderate ADLs, and frailty (Table 1). Our study indicated that anxiety and depression were associated with multivariate factors except bone mineral density(BMD)in the elderly; the results are consistent with other study findings [33]. A previous study showed that approximately $45 \%$ of women with depression had $\mathrm{OP}$, the duration of depression was strongly negatively associated with BMD, and depression reduced physical activity [32]. Another study also showed that BMD was negatively associated with depressive symptoms in older patients [34], and depression affected the patient's mood and increased comorbidities, disabilities, and medical resources [32]. 
Our study showed that osteoporotic fracture was only associated with anxiety and had no significant effect on depression; this result is inconsistent with other study findings [35]. Although the prevalence of osteoporotic fractures (35.3\%) was higher in this study, patients had a good prognosis after fracture treatment, and their quality of life was not affected. Income affected depression but not anxiety in our study. The possible reason is that these elderly people had to pay their medical expenses and daily living expenses, which affected their mood. We found that pain affected the anxiety and depression of participants; this finding is consistent with another study [36]. Chronic pain can result in mobility restrictions that interfere with daily activities and cause depression [37]. The frequency of participation in social activity is a factor influencing anxiety and depression. On the one hand, participants lacked energy because of OP; on the other hand, this may be related to our selection time. This research occurred during the global COVID-19 pandemic, and people were restricted from traveling and participating in parties or social activity, which may affect the survey results. The prevalence of anxiety and depression in the elderly without occupation was higher than that in the elderly with occupation. The reason may be that their social circle was small, which affected their mood. Participants who lived with a child not only supported their children financially but also helped them look after their grandchildren. Financial and physical stress led to anxiety and depression. This study found that comorbid medical conditions and multiple medications were closely related to anxiety and depression; this result is consistent with a previous study [38].

Our research showed that increasing age, pain, $\geq 5$ types of drugs, no participation in social activity, heavy ADLs, frailty, severe anxiety, and severe depression decreased PA (Table 2). The reasons may be due to low body energy because of disease, drug side effects, and mood. The participants who were divorced/widowed had decreased PA because of a lack of family member support. Participants whose education was primary school and below and who had no occupation had reduced PA and might have lacked OP-related knowledge. For participants whose income was supported by their child and who lived with children, reduced PA may be due to time and low body energy. Studies have reported that PA and exercise can be used as primary or adjunctive therapies to prevent and treat OP, especially in elderly individuals $[39,40]$.

Osteoporosis is an age-related disease, and smoking is associated with a higher risk of osteoporotic fractures [41]. Participation in social activity, ADLs, SAS, and SDS were the main risk factors that affected PA in elderly patients with OP (Table 3); the results are consistent with a relevant study [42]. The prevalence of frailty was higher among our participants than among people aged $\geq 60$ years in China (prefrail: $20.8 \%$, frail: $71.7 \%$ vs prefrail: $51.2 \%$, frail: $7.0 \%$ ) [43]. OP and frailty have similar risk factors which interact to cause dysfunction, such as malnutrition, poor muscle strength, low body energy, increased risks of falls, fractures, comorbidity, disability, and mortality [44-46], and decreased resilience to stress or events and ADLs [43].

The present study showed that physical activity is negatively related to anxiety and depression in elderly outpatients with OP. This result is consistent with other literature suggesting that the relationship between depression and PA in OP is bidirectional [47-49] (Fig. 2). Physiological changes in BMD and depressive 
states in elderly patients and patients with OP force them to change their daily lifestyles. For example, to prevent falls and avoid fractures, patients are afraid to participate in their favorite exercises, which can negatively impact their mood [50]. PA intervention can reduce anxiety and depression in older adults and the impaired function caused by OP [51]. Another study reported the negative effects of antidepressant drugs and antianxiety drugs on OP and adverse reactions to these drugs, such as dizziness and headaches, which will also lead to reduced PA. On the other hand, patients with anxiety and depression have reduced PA because they are reluctant to participate in social activity $[49,51]$. In summary, these changes will increase the prevalence of anxiety and depression in elderly individuals with OP.

\section{Conclusions}

We found that anxiety and depression were prevalent and that physical activity was negatively correlated with anxiety and depression in elderly outpatients with OP in China. Age, education, smoking history, participation in social activity, ADLs, frailty, SAS, and SDS were the main risk factors for PA in elderly patients with OP. Therefore, medical staff should pay attention to screening OP in the elderly, identify the influencing factors of anxiety and depression in time, and reduce the incidence of anxiety and depression. At the same time, we should encourage the elderly to engage in physical exercise in their daily lives. Further research on this topic is required to gain deeper insight into these relationships.

\section{Limitations}

Our study had some limitations that need to be considered. The main limitation of our study is a convenience sample, but not having a randomized sampling method may have resulted in a small sample size if we consider that we are referring to elderly patients with OP in China. At the same time, the lack of a control group limits the generalization of the results. Another limitation relates to the participants' recruitment, in which the proportion of female patients (83.20\%) was higher than that of male patients $(16.80 \%)$. However, it should be noted that this limitation constitutes a methodological problem in this study. A previous study showed that depression risk in female patients was associated with the diagnosis of OP [35]. It may not be possible to generalize these study results to other cultures and geographic regions in China.

\section{Abbreviations}

DEXA: dual-energy X-ray absorptiometry devices, OP: Osteoporosis,

PA: physical activity, BMD: bone mineral density,

SAS: Self-rating Anxiety Scale, SDS: Self-rating Depression Scale,

PASE: Physical Activity Scale in the Elderly, BMI: body mass index,

ADL: Activity of Daily Life 


\section{Declarations}

\section{Ethics approval and consent to participate}

The ethics committee of the West China Hospital of Sichuan University approved this study and its methods in December 2020 (approve number 2020 (59)). All participants provided signed informed consent.

\section{Consent for publication}

Not applicable.

\section{Availability of data and materials}

The datasets used and analyzed during the current study are available from the corresponding author on reasonable request.

\section{Competing interests}

The authors declare that they have no competing interests.

\section{Funding}

1. West China Nursing Discipline Development Special Fund Project, Sichuan University, China, NO: HXHL19021.

2. Science and Technology Department of Sichuan Province, China, NO: 2020YFS0151.

\section{Authors' contributions}

FR carried out conceptualization, project administration and supervision. ZX, LW, GY and SL performed the investigation and prepared the original draft. DX analyzed and interpreted the data. FR and SL reviewed and edited the manuscript. All authors read and approved the final manuscript.

\section{Acknowledgment}

We are grateful to all patients with OP who participated in the study.

\section{Author details}

1,2,3,4 and* West China School of Nursing/Department of International Medical Center, West China Hospital, Sichuan University,Sichuan,China.5 West China School of Nursing, Sichuan University,Sichuan, China.

\section{Statement}


All methods were carried out in accordance with relevant guidelines and regulations.

\section{References}

1. Seriolo B, Paolino S, Casabella A, Botticella G, Seriolo C, Molfetta L.Osteoporosis in the elderly. Aging Clin Exp Res. 2013,25 Suppl 1:S27-9.

2. Choi HJ, Shin CS, Ha YC, Jang S, Jang SY, Park C, et al. Burden of osteoporosis in adults in Korea:a national health insurance database study.Journal of Bone and Mineral Metabolism. 2012,30(1):548.

3. Lee SR, Ha YC, Kang H, Park YG, Nam KW, Kim SR..Morbidity and mortality in Jeju residents over 50years of age with hip fracture with mean 6-year follow-up:a prospective cohort study.J Korean Med Sci. 2013,28(7):1089-94.

4. Lin $X$, Xiong $D$, Peng YQ, Sheng $Z F, W u X Y, W u X P$, et al. Epidemiology and management of osteoporosis in the People's Republic of China:current perspectives. Clinical interventions in aging.2015,10:1017-33.

5. Si L, Winzenberg TM, Jiang Q, Chen M, Palmer AJ. Projection of OP related fractures and costs in China: 2010-2050. Osteoporos Int.2015,26(7) :1929-37.

6. He LY, Sun Y, Yao WJ, Pan KQ. The prevalence rate of osteoporosis in the elderly in China between 2010 and 2016.A Meta-analysis of single rate.Chinese Journal of Osteoporosis.2016, 22(12):1590-6. [Chinese]

7. Johnell 0 , Kanis JA.An estimate of the worldwide prevalence and disability associated with osteoporotic fractures. Osteoporos Int.2006,17(12):1726-33.

8. Papaioannou A, Morin S, Cheung AM, Atkinson S, Brown JP, Feldman S, et al.Scientific Advisory Council of OP Canada. 2010 clinical practice guidelines for the diagnosis and management of OP in Canada: summary. CMAJ. 2010,182(17): 1864-73.

9. Kanis JA, McCloskey EV, Johansson H, Cooper C, Rizzoli R, Reginster JY. Scientific Advisory Board of the European Society for Clinical and Economic Aspects of Osteoporosis and Osteoarthritis (ESCEO) and the Committee of Scientific Advisors of the International Osteoporosis Foundation (IOF). European guidance for the diagnosis and management of osteoporosis in postmenopausal women. Osteoporos Int. 2013,24(1):23-57.

10. Lin SF, Li YZ. The treatment rate and compliance of anti-osteoporosis drugs.Chinese Journal of osteoporosis.2020,26(3):439-44.[Chinese]

11. Wang $\mathrm{O}, \mathrm{Hu} Y$, Gong S, Xue Q, Deng Z, Wang L, et al. A survey of outcomes and management of patients post fragility fractures in China. Osteoporos Int.2015,26(11):2631-40.

12. Yamazaki S, Ichimura S, Iwamoto J, Takeda T, Toyama Y. Effect of walking exercise on bone metabolism inpostmenopausal women with osteopenia/osteoporosis.Journal of Bone and Mineral Metabolism.2004,22(5):500-8. 
13. Caputo Eduardo Lucia,Costa Marcelo Zanusso.Influence of physical activity on quality of life in postmenopausal women with osteoporosis. Revista Brasileira de Reumatologia.2014,54(6): 467-73.

14. Park KS, Yoo JI, Kim HY, Jang S, Park Y, Ha YC.Education and exercise program improves osteoporosis knowledge and changes calcium and vitamin D dietary intake in community dwelling elderly.BMC Public Health.2017,17(1):966.

15. Daly RM, Dalla VJ, Duckham RL, Fraser SF, Helge EW. Exercise for the prevention of osteoporosis in postmenopausal women:an evidence-based guide to the optimal prescription. Brazilian Journal of Physical Therapy.2019,23(2):170-80.

16. Cawthon PM, Fullman RL, Marshall L, Mackey DC, Fink HA, Cauley JA, et al. Osteoporotic Fractures in Men Research G.Physical performance and risk of hip fractures in older men. J Bone Miner Res.2008,23(7):1037-44.

17. Melton LJ 3rd. Epidemiology worldwide. Endocrinol Metab Clin North Am. 2003,32(1):1-13.

18. Piscitelli P, Metozzi A, Benvenuti E, Bonamassa L, Brandi G, Cavalli L, et al. Connections between the outcomes of osteoporotic hip fractures and depression, delirium or dementia in elderly patients: rationale and preliminary data from the CODE study. Clin Cases Miner Bone Metab. 2012,9(1):40-4.

19. Drosselmeyer J, Rapp MA, Hadji P, Kostev K. Depression risk in female patients with osteoporosis in primary care practices in Germany.Osteoporos Int.2016,27(9):2739-44.

20. Ma YZ, Wang YP, Liu Q, Li CL, Ma X, Wang YJ, et al. 2018 China guideline for diagnosis and treatment of senile osteoporosis.Chin J Osteoporos.2018,24(12):1541-67.[Chinese]

21. MAHONEY FI, BARTHEL DW. FUNCTIONAL EVALUATION: THE BARTHEL INDEX. Md State Med J.1965,14:61-5.

22. Fried LP, Tangen CM, Walston J, Newman AB, Hirsch C, Gottdiener J, et al. Frailty in older adults:evidence for a phenotype.J Gerontol Med Sci.2001,56A(3):M146-56.

23. Zung,W.W.K. A Rating instrument for Anxiety Disorders.Psychosomatics.1971,12:371-379.

24. Dunstan DA, Scott N, Todd AK. Screening for anxiety and depression:reassessing the utility of the Zung scales.BMC Psychiatry.2017,17(1):329.

25. Sharpley CF, Christie DR. An analysis of the psychometric profile and frequency of anxiety and depression in Australian men with prostate cancer. Psychooncology. 2007,16(7):660-7.

26. Dunstan DA, Scott N. Norms for Zung's self-rating anxiety scale.BMC Psychiatry.2020, 20(1):1-8.

27. Zung,W.W.K. A self-rating depression scale.Archives of General Psychiatry.1965,12: 63-70.

28. Biggs JT, Wylie L, Ziegler VE. Validity of the Zung self rating depression Scale. British Journal of Psychiatry.1978,132(4):381-5.

29. Washburn RA, Smith KW, Jette AM, Janney CA.The physical activity scale for the elderly (PASE):development and evaluation.J Clin Epidemiol.1993,46(2):153-162.

30. Hu B, Lin LF, Zhuang MQ, Yuan ZY, Li SY, Yang YJ, et al. Reliability and relative validity of three physical activity questionnaires in Taizhou population of China:the Taizhou Longitudinal Study.Public Health.2015,129(9):1211-7. 
31. Tao YX.The using of Chinese version of Physical Activity Scale for the Elderly in old patients with COPD and analysis the influencing factors of physical activity [D].Tianjin medical university.2017,19. [Chinese].

32. Petronijević M, Petronijević N, Ivković M, Stefanović D, Radonjić N, Glisić B, et al. Low bone mineral density and high bone metabolism turnover in premenopausal women with uni polar depression.Bone.2008,42(3):582-90.

33. Erez HB, Weller A, Vaisman N, Kreitler S. The relationship of depression, anxiety and stress with low bone mineral density in post-menopausal women.Arch Osteoporos. 2012,7:247-55.

34. Robbins J, Hirsch C, Whitmer R, Cauley J, Harris T. The association of bone mineral density and depression in an older population.J Am Geriatr Soc.2001,49:732-6.

35. Reid KJ, Harker J, Bala MM, Truyers C, Kellen E, Bekkering GE. Epidemiology of chronic non-cancer pain in Europe:narrative review of prevalence, pain treatments and pain impact.Curr Med Res Opin.2011,27(2):449-62.

36. Maske UE, Busch MA, Jacobi F, Riedel-Heller SG, Scheidt-Nave C, Hapke U. Chronic somatic conditions and mental health problems in the general population in Germany. Results of the national telephone health interview survey "German health update (GEDA)"2010].Psychiatr Prax. 2013,40(4):207-13.[German]

37. Bianchi ML, Orsini MR, Saraifoger S, Ortolani S, Radaelli G, Betti S. Quality of life in post-menopausal osteoporosis. Health Qual Life Outcomes. 2005, 1,3:78.

38. McMillan LB, Zengin A, Ebeling PR, Scott D. Prescribing Physical Activity for the Prevention and Treatment of Osteoporosis in Older Adults. Healthcare (Basel). 2017,5(4):85.

39. Bijelic R, Milicevic S, Balaban J. Risk Factors for Osteoporosis in Postmenopausal Women. Med Arch.2017,71(1):25-8.

40. Korkor AB, Eastwood D, Bretzmman C. Effects of gender, alcohol, smoking, and dairy consumption on bone mass in Winsconsin adolescents.WMJ. 2009,108(4): 181-8.

41. You Y, Meng X, Qiu HY. Depressive symptoms,obesity and functional disability among middle -aged and older adults in China:empirical evidence based on CHARLS.Modern Preventive Medicine.2019,46(19):3680-84.[Chinese]

42. Wu C, Smit E, Xue QL, Odden MC. Prevalence and correlates of frailty among community-dwelling Chinese older adults: the China health and retirement longitudinal study.J Gerontol A Biol Sci Med Sci.2017,73(1):102-8.

43. Khosla S, Oursler MJ, Monroe DG. Estrogen and the skeleton. Trends Endocrinol Metab. 2012,23(11):576-81.

44. Hanlon P, Nicholl BI, Jani BD, Lee D, McQueenie R, Mair FS. Frailty and prefrailty in middle-aged and older adults and its association with multi-morbidity and mortality: a prospective analysis of 493737 UK biobank participants.Lancet Public Health. 2018,3(7): E323-32.

45. Gu D, Feng Q. Frailty still matters to health and survival in centenarians: the case of China. BMC Geriatr.2015,15:159. 
46. Mezuk B, Eaton WW, Golden SH, Golden SE. Depression and Osteoporosis: epidemiology and potential mediating pathways. Osteoporos Int.2008,19(1):1-12.

47. Duncan R, Turner $\mathrm{CH}$. Mechanotransduction and the Functional Response of Bone to Mechanical Strain.Calcif Tissue Int.1995,57(5):344-58.

48. Erez HB, Weller A, Vaisman N, Kreitler S. The relationship of depression, anxiety and stress with low bone mineral density in post-menopausal women.Arch Osteoporos. 2012,7:247-55.

49. Lips P, Agnusdei D, Caulin F, Cooper C, Johnell O, Kanis J, et al. The development of a European questionnaire for quality of life in patients with vertebral osteoporosis.Scand $\mathrm{J}$ Rheumatol.1996,103:84-5.

50. Aguiñaga S, Ehlers DK, Salerno EA, Fanning J, Motl RW, McAuley E. Home-Based Physical Activity Program Improves Depression and Anxiety in Older Adults.J Phys Act Health. 2018, 15(9):692-6.

51. Cauley JA, Fullman RL, Stone KL, Zmuda JM, Bauer DC, Barrett-Connor E, et al. Factors associated with the lumbar spine and proximal femur bone mineral density in older men. Osteoporos Int.2005,16:1525-37.

\section{Tables}

Due to technical limitations, table 1 and 2 are only available as a download in the Supplemental Files section.

\section{Figures}
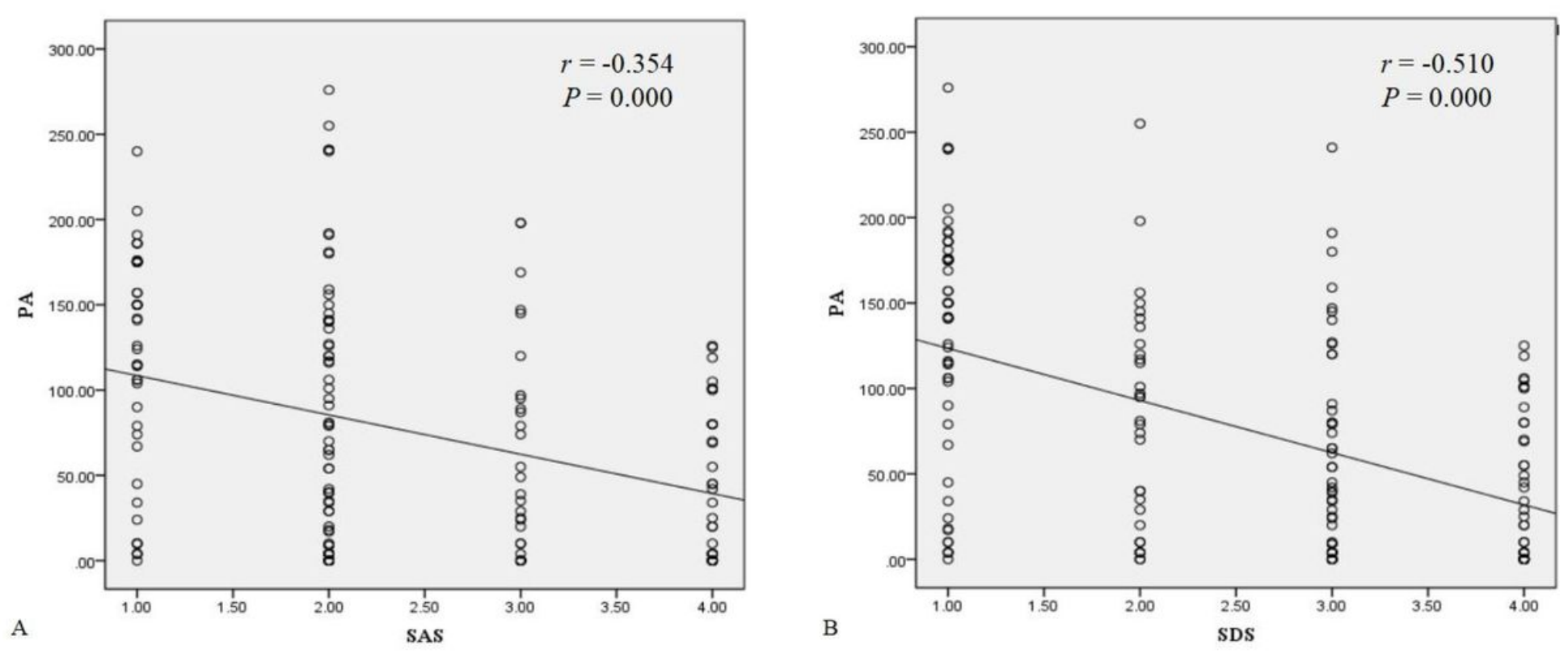

\section{Figure 1}

Correlation between physical activity and anxiety and depression, $n=173$ 


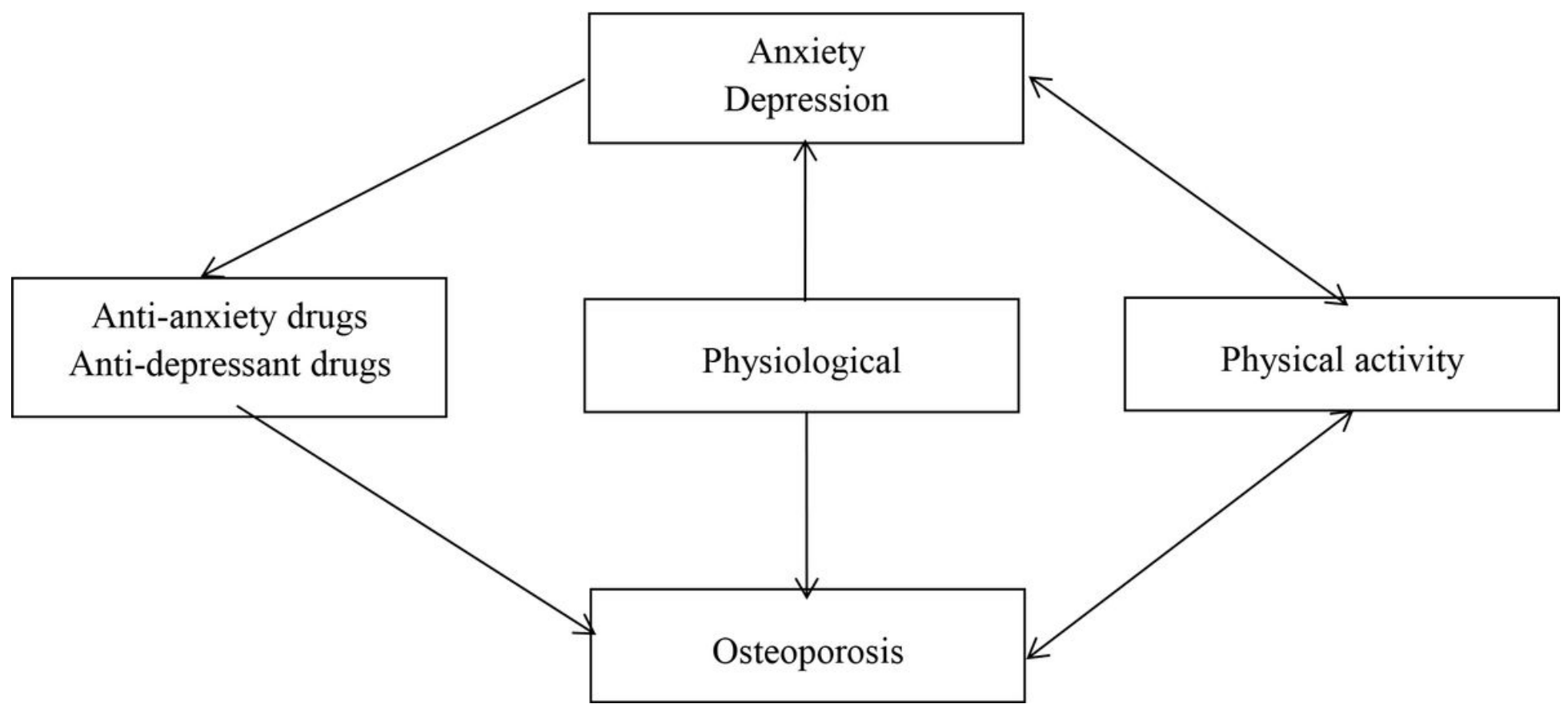

Figure 2

The bidirectional relationship of anxiety, depression, and PA in OP [46-48]

\section{Supplementary Files}

This is a list of supplementary files associated with this preprint. Click to download.

- Table1.xIs

- Table2.xls 